\title{
A QUARANTINE PROTOCOL AGAINST RED PALM WEEVIL RHYNCHOPHORUS FERRUGINEUS (OLIVIER) (COLEPTERA: CURCULIONDAE) IN DATE PALM
}

\author{
Abdul Moneim Al-Shawaf ${ }^{1}$, Abdullah Al-Shagag ${ }^{1}$, Mansour Al-Bagshi, Sami Al-Saroj ${ }^{1}$, \\ Salim Al-Bather ${ }^{1}$, Abdel Moneim Al-Dandan ${ }^{1}$, Abdallah Ben Abdallah ${ }^{2 *}$, Jose Romeno Faleiro ${ }^{2}$
}

\author{
${ }^{1}$ Date Palm Research Centre (DPRC), P.O. Box 43, Al-Hassa-31982, Kingdom of Saudi Arabia \\ ${ }^{2}$ FAO Project (UTFN/SAU/043/SAU), DPRC, P.O. Box 43, Al-Hassa-31982, Kingdom of Saudi Arabia
}

Received: March 31, 2013

Accepted: October 24, 2013

\begin{abstract}
The red palm weevil (RPW) Rhynchophorus ferrugineus (Olivier), (Coleptera: Curculiondae) is a key pest of date palm in the Middle East. This weevil is currently being reported from over $50 \%$ of the date growing countries of the world. The date palm Phoenix dactylifera cropped area in the Middle East, has significantly increased during the last two decades where date palm is mainly propagated through offshoots. The red palm weevil larvae are often found in the offshoots, resulting in the spread of the pest and also its re-inoculation where RPW is already controlled. Currently, there are no quarantine protocols to ensure that date palm offshoots which are transported for planting, are free of RPW larvae. In this study, date palm offshoots were sprayed while still attached to the mother palm and also dipped separately with Fipronil 3.5\% (Thripguard $35 \mathrm{SC}^{\mathrm{TM}}$ ) and Imidacloprid 35\% (Confidor $350 \mathrm{SC}^{\mathrm{TM}}$ ) a day after detachment from the mother palm. Fipronil and Imidacloprid were tested at $0.008 \%$ and $0.01 \%$, respectively. Results revealed that dipping gave complete mortality of the larvae. Dipping was significantly better than spraying since spraying resulted in only partial mortality of the larvae. It was also seen that dipping offshoots in Fipronil $0.004 \%$ and $0.002 \%$ for 30 and 60 min, respectively, resulted in $100 \%$ mortality of the larvae tested. Further, toxicity studies resulted in complete mortality of larvae and adults that were exposed to offshoot tissue that was dipped in Fipronil at the above concentrations. For this reason, it is recommended to dip date palm offshoots in $0.004 \%$ Fipronil for 30 min before transporting to ensure complete mortality of the hidden larval stages, if any and complete certification and transport of the treated offshoots to the new planting site within $72 \mathrm{~h}$ of treatment.
\end{abstract}

Key words: date palm offshoots, Fipronil, Imidacloprid, insecticides, quarantine, red palm weevil

\section{INTRODUCTION}

The date palm, Phoenix dactylifera L., is closely associated with the life of the people in the Gulf region of the Middle East. This region accounts for about $30 \%$ of the world's date production, with Saudi Arabia and the United Arab Emirates being the region's top producers (FAOstat 2012). The crop has a high socioeconomic importance not only due to its food value, but also its capacity to provide many other products such as shelter, fiber, clothing, aesthetic beauty, and furniture (Mousavi et al. 2009).

The red palm weevil (RPW), Rhynchophorus ferrugineus (Olivier) (Coleoptera: Curculionidae), has become a key pest of date palm and has been reported in over $50 \%$ of the date growing countries worldwide (Faleiro 2006). In the Middle East, RPW was reported from Rass-El-Khaima in the United Arab Emirates, in 1985. It then spread to all the countries in the Gulf region infesting 5 to $6 \%$ of palms in the region (Zaid et al. 2002). The annual economic loss due to eradication of the severely infested palms in the Gulf countries in which those countries had between 1 and $5 \%$ infestation, has been estimated to range from
$\$ 5.18$ to $\$ 25.92$ million, respectively (El-Sabea et al. 2009). In the Mediterranean basin, RPW spread slowly during the mid-1990s and rapidly from 2004 onwards. In the Mediterranean basin, it is a key pest of Phoenix canariensis hort. ex Chabaud (Dembilio et al. 2009). Currently, RPW is reported to infest 40 palm species worldwide (OJEU 2008, Malumphy and Moran 2009; EPPO 2009; Anonymous 2013) with a wide ecological range in all the continents of the world. Recently, Fiaboe et al. 2012 have predicted the spread of the pest to new regions of the world using ecological niche modeling.

The Food and Agriculture Organization of the UN has identified RPW as a category-1 pest of date palm in the Middle East. The red palm weevil is reported to mostly attack young date palms less than 20 years old. Current management of the weezil is mainly through a pheromone based Integrated Pest Management (IPM) strategy. Early detection of infestation in the field is important to the success of any RPW-IPM programme (Abraham et al. 1998; Oehlschlager 2005). Recently, an inspection of nearly 40,000 date palms in the Al-Hassa oasis of Saudi 
Arabia took place. There were found to be 167 infestations of which $78 \%$ were in the early stage of the attack and were treated with insecticide (Sallam et al. 2012). Such infestations usually harbor RPW larvae that are eliminated with insecticide administered as a trunk injection (Faleiro 2006). In Egypt, quarantine and certification programmes recommend zero tolerance for RPW so as to block the spread of the pest to secondary foci within planted acreage (Salama and Abd-Elgawad 2003). Date palm is mainly propagated through offshoots which often harbour larval stages of RPW. In the early instars, the RPW larval stages are extremely difficult to detect due to lack of any visual symptoms of damage that are associated with the later larval instars. A RPW infested date palm exhibits several symptoms depending on the stage of attack viz. oozing of brownish fluid together with palm tissue excreted by feeding of larvae which has a typical fermented odor, drying of infested offshoots, tunneling of palm tissue by larvae, presence of adults and pupae at the base of fronds, fallen pupae around an infested palm, drying of outer leaves and fruit bunches and toppling of the trunk in case of very severe and extensive tissue damage (Abraham et al. 1998).

Countries in the Middle East have issued federal decrees that restrict movement of palms, including date palm offshoots, in the region, due to the threat of RPW. Ideally, movement of planting material from infested acreage within the country and also from one country to another, needs to be stopped. Wherever this is not possible it is essential to implement strict pre- and post-entry quarantine regimes, wherein only pest free and certified planting material can be transported. Insecticide based on quarantine protocols have yet to be standardized for adoption in the field with respect to transport/shipping of planting material, especially date palm (Faleiro 2006). There are no reports on quarantine treatments that ensure movement of RPW-free date palm offshoots. We tested and developed an insecticide based protocol that kills larval stages of RPW feeding inside date palm offshoots.

\section{MATERIALS AND METHODS}

The trials to develop insecticide based quarantine protocols against larval stages infesting date palm offshoots, were carried out using 3-5 year old offshoots of the premier Saudi Arabian date palm variety 'Khalas'. The trials were carried out at the Ministry of Agriculture's Date Palm Research Centre, Al Hassa, Saudi Arabia, under the following sequence.

\section{Treatment method and insecticide efficacy}

In this trial, there were three treatment methods (factor A) and two insecticides (factor B).

The treatments in factor A were (i) dipping the bole of date palm offshoots in insecticide solution for $6 \mathrm{~h}$ with holes to the bole of the offshoot, (ii) dipping date palm offshoots in insecticide solution for $6 \mathrm{~h}$ without holes to the bole of the offshoot and (iii) spraying the offshoots with insecticides at $10 \mathrm{~L} /$ offshoot. The treatments in factor B were (i) Fipronil 3.5\% (Thripgaurd $35 \mathrm{SC}^{\mathrm{TM}}$ ) at a concen- tration of $0.008 \%$ i.e. $2.3 \mathrm{ml} / \mathrm{L}$, and (ii) Imidacloprid 35\% (Confidor $350 \mathrm{SC}^{\mathrm{TM}}$ ) at a concentration of $0.01 \%$ i.e. 0.3 $\mathrm{ml} / \mathrm{L}$. Fipronil is a broad-spectrum systemic phenylpyrazole insecticide, while Imidacloprid is a systemic neonicotinoid insecticide in the chloronicotinyl nitroguanidine chemical family (Tomlin 2011).

To test for better absorption of the insecticides during the dipping, five slanting holes $(6 \mathrm{~mm}$ wide and $15 \mathrm{~cm}$ deep) were drilled equidistantly into each offshoot in one treatment set while also dipping offshoots without any holes in another treatment set. The untreated control was maintained by dipping/spraying the offshoots with water.

After the dipping treatment, offshoots were placed standing/upright overnight to drain off the excess solution (insecticide/water) before inoculating with RPW larvae. In the spray treatment, offshoots were detached carefully from the mother palms after $24 \mathrm{~h}$ of spraying, and brought to the laboratory for inoculation with RPW larvae.

All the offshoots in the trial were split into two equal longitudinal halves. Additionally, two chambers $(15 \times 10 \times$ $\times 3 \mathrm{~cm}$ ) were prepared in one half of the offshoot. Each chamber was separately inoculated with three small (average weight of one larva: $0.20 \mathrm{~g}$ ), and two medium (average weight of one larva: $0.70 \mathrm{~g}$ ) RPW larvae that were previously starved for two hours. Subsequently, the other half of the offshoot was placed over the half inoculated with RPW larvae. Both halves of the offshoots were secured over one another with pliable wire. This trial was carried out twice using the two factor randomized block design wherein each treatment was replicated five times.

Observations on larval mortality were recorded $24 \mathrm{~h}$ after inoculation (48 $\mathrm{h}$ after dipping/spraying).

\section{Standardize the concentration of Fipronil and duration of treatment}

From trial 1, it was seen that dipping offshoots in Fipronil $0.008 \%$ for six hours without making holes to the bole of the offshoot, gave the best result. The next stage in the trial was to reduce both the concentration of Fipronil and also the time required for treatment. We tested three concentrations (factor A) viz. 0.004, 0.002, 0.001\%, each of which was tested for 60, 30, and 15 min (factor B), using the dipping protocol (without drilling any hole) adopted in the first stage of the experiment described above. This trial was also carried out using the two factor randomized block design. Five replications were maintained for each treatment.

\section{Test residual toxicity of insecticides against RPW}

To assess the residual toxicity of the tested insecticides, a larva each (average weight of one larva: small $0.20 \mathrm{~g}$, medium $-0.70 \mathrm{~g}$, large $-2.24 \mathrm{~g}$ ) and two newly emerged adults (male/female) were caged with $200 \mathrm{~g}$ of palm tissue scooped out of the treated (dipped/sprayed/ the control) offshoots, $72 \mathrm{~h}$ after dipping and $24 \mathrm{~h}$ after spraying. Subsequently, observations on larval/adult mortality were recorded after 4 days of treatment. Each treatment was replicated five times in the above trials. Similar residual toxicity studies were also carried out for the second trial involving the best treatment. 
In all the above trials, data on mean mortality was subjected to analysis of variance (ANOVA). Results pertaining to the above trials are presented below.

Ten date palm offshoots treated with the recommended quarantine protocol were planted in the farm of the Date Palm Research Centre, Al-Hassa, Saudi Arabia, to record any phytotoxicity symptoms, during a period of one year, along with the untreated control and offshoots dipped in water only.

\section{RESULTS}

As regards the method of treatment, results on mean larval mortality presented in table 1 indicate that dipping date palm offshoots in insecticide was better than spraying. Also, statistically similar mortality levels were obtained in offshoots dipped in insecticide with and without holes. So, the indication is, that drilling holes in offshoots before dipping in insecticide is not required. Table 1 reveals that Fipronil is superior to Imidacloprid.

Table 1. Mortality of R. ferrugineus larvae in date palm offshoots treated with insecticide

\begin{tabular}{|c|c|c|c|c|c|c|}
\hline \multirow{3}{*}{ Treatment } & \multicolumn{6}{|c|}{ Mean larval mortality [\%] ${ }^{*}$} \\
\hline & \multicolumn{2}{|c|}{ small larvae } & \multicolumn{2}{|c|}{ medium larvae } & \multicolumn{2}{|c|}{ cumulative analysis } \\
\hline & trial-i & trial-ii & trial-i & trial-ii & small larvae & medium larvae \\
\hline \multicolumn{7}{|c|}{ Factor A: Treatment method (Dipping/Spraying) } \\
\hline $\mathrm{A} 1$ & $\begin{array}{c}6.30 \\
(60.00)\end{array}$ & $\begin{array}{c}6.92 \\
(66.67)\end{array}$ & $\begin{array}{c}5.06 \\
(46.67)\end{array}$ & $\begin{array}{c}6.53 \\
(60.00)\end{array}$ & $\begin{array}{c}6.72 \\
(63.33)\end{array}$ & $\begin{array}{c}6.11 \\
(53.33)\end{array}$ \\
\hline A2 & $\begin{array}{c}6.30 \\
(60.00)\end{array}$ & $\begin{array}{c}6.36 \\
(57.78)\end{array}$ & $\begin{array}{c}6.10 \\
(56.67)\end{array}$ & $\begin{array}{c}6.53 \\
(60.00)\end{array}$ & $\begin{array}{c}6.48 \\
(58.89)\end{array}$ & $\begin{array}{c}6.46 \\
(58.33)\end{array}$ \\
\hline $\mathrm{A} 3$ & $\begin{array}{c}1.73 \\
(6.67)\end{array}$ & $\begin{array}{c}2.07 \\
(8.89)\end{array}$ & $\begin{array}{c}0.71 \\
(0.00)\end{array}$ & $\begin{array}{c}0.71 \\
(0.00)\end{array}$ & $\begin{array}{c}2.31 \\
(7.78)\end{array}$ & $\begin{array}{c}0.71 \\
(0.00)\end{array}$ \\
\hline $\mathrm{CD}(\mathrm{p}=0.05)$ & 1.71 & 1.02 & 1.56 & 0.52 & 0.71 & 0.52 \\
\hline \multicolumn{7}{|c|}{ Factor B: Insecticide (Fipronil/Imidacloprid) } \\
\hline B1 & $\begin{array}{c}7.60 \\
(71.11)\end{array}$ & $\begin{array}{c}7.60 \\
(71.11)\end{array}$ & $\begin{array}{c}6.92 \\
(66.67)\end{array}$ & $\begin{array}{c}6.92 \\
(66.67)\end{array}$ & $\begin{array}{c}7.84 \\
(71.11)\end{array}$ & $\begin{array}{c}6.92 \\
(66.67)\end{array}$ \\
\hline B2 & $\begin{array}{c}6.02 \\
(55.56)\end{array}$ & $\begin{array}{c}7.04 \\
(62.22)\end{array}$ & $\begin{array}{c}4.24 \\
(56.67)\end{array}$ & $\begin{array}{c}6.14 \\
(53.33)\end{array}$ & $\begin{array}{c}6.97 \\
(58.89)\end{array}$ & $\begin{array}{c}5.65 \\
(45.00)\end{array}$ \\
\hline B3 & $\begin{array}{c}0.71 \\
(0.00)\end{array}$ & $\begin{array}{c}0.71 \\
(0.00)\end{array}$ & $\begin{array}{c}0.71 \\
(0.00)\end{array}$ & $\begin{array}{c}0.71 \\
(0.00)\end{array}$ & $\begin{array}{c}0.71 \\
(0.00)\end{array}$ & $\begin{array}{c}0.71 \\
(0.00)\end{array}$ \\
\hline $\mathrm{CD}(\mathrm{p}=0.05)$ & 1.71 & 0.52 & 1.56 & 0.52 & 0.71 & 0.52 \\
\hline \multicolumn{7}{|c|}{ Interaction $(\mathrm{A} \times \mathrm{B})$} \\
\hline A1B1 & $\begin{array}{c}10.03 \\
(100.00)\end{array}$ & $\begin{array}{c}10.03 \\
(100.00)\end{array}$ & $\begin{array}{c}10.03 \\
(100.00)\end{array}$ & $\begin{array}{c}10.03 \\
(100.00)\end{array}$ & $\begin{array}{c}10.03 \\
(100.00)\end{array}$ & $\begin{array}{c}10.03 \\
(100.00)\end{array}$ \\
\hline A1B2 & $\begin{array}{c}8.16 \\
(80.00)\end{array}$ & $\begin{array}{c}10.03 \\
(100.00)\end{array}$ & $\begin{array}{c}4.43 \\
(40.00)\end{array}$ & $\begin{array}{c}8.86 \\
(80.00)\end{array}$ & $\begin{array}{c}9.44 \\
(90.00)\end{array}$ & $\begin{array}{c}7.60 \\
(60.00)\end{array}$ \\
\hline A1B3 & $\begin{array}{c}0.71 \\
(0.00)\end{array}$ & $\begin{array}{c}0.71 \\
(0.00)\end{array}$ & $\begin{array}{c}0.71 \\
(0.00)\end{array}$ & $\begin{array}{c}0.71 \\
(0.00)\end{array}$ & $\begin{array}{c}0.71 \\
(0.00)\end{array}$ & $\begin{array}{c}0.71 \\
(0.00)\end{array}$ \\
\hline A2B1 & $\begin{array}{c}10.03 \\
(100.00)\end{array}$ & $\begin{array}{c}10.03 \\
(100.00)\end{array}$ & $\begin{array}{c}10.03 \\
(100.00)\end{array}$ & $\begin{array}{c}10.03 \\
(100.00)\end{array}$ & $\begin{array}{c}10.03 \\
(100.00)\end{array}$ & $\begin{array}{c}10.03 \\
(100.00)\end{array}$ \\
\hline A2B2 & $\begin{array}{c}8.61 \\
(80.00)\end{array}$ & $\begin{array}{c}8.34 \\
(73.33)\end{array}$ & $\begin{array}{c}7.58 \\
(70.00)\end{array}$ & $\begin{array}{c}8.86 \\
(80.00)\end{array}$ & $\begin{array}{c}8.71 \\
(76.67)\end{array}$ & $\begin{array}{c}8.64 \\
(75.00)\end{array}$ \\
\hline A2B3 & $\begin{array}{c}0.71 \\
(0.00)\end{array}$ & $\begin{array}{c}0.71 \\
(0.00)\end{array}$ & $\begin{array}{c}0.71 \\
(0.00)\end{array}$ & $\begin{array}{c}0.71 \\
(0.00)\end{array}$ & $\begin{array}{c}0.71 \\
(0.00)\end{array}$ & $\begin{array}{c}0.71 \\
(0.00)\end{array}$ \\
\hline A3B1 & $\begin{array}{c}2.75 \\
(13.33)\end{array}$ & $\begin{array}{c}2.75 \\
(13.33)\end{array}$ & $\begin{array}{c}0.71 \\
(0.00)\end{array}$ & $\begin{array}{c}0.71 \\
(0.00)\end{array}$ & $\begin{array}{c}3.46 \\
(13.34)\end{array}$ & $\begin{array}{c}0.71 \\
(0.00)\end{array}$ \\
\hline A3B2 & $\begin{array}{c}1.73 \\
(6.67)\end{array}$ & $\begin{array}{c}2.75 \\
(13.33)\end{array}$ & $\begin{array}{c}0.71 \\
(0.00)\end{array}$ & $\begin{array}{c}0.71 \\
(0.00)\end{array}$ & $\begin{array}{c}2.77 \\
(10.00)\end{array}$ & $\begin{array}{c}0.71 \\
(0.00)\end{array}$ \\
\hline A3B3 & $\begin{array}{c}0.71 \\
(0.00) \\
\end{array}$ & $\begin{array}{c}0.71 \\
(0.00)\end{array}$ & $\begin{array}{c}0.71 \\
(0.00)\end{array}$ & $\begin{array}{c}0.71 \\
(0.00) \\
\end{array}$ & $\begin{array}{c}0.71 \\
(0.00)\end{array}$ & $\begin{array}{c}0.71 \\
(0.00) \\
\end{array}$ \\
\hline $\mathrm{CD}(\mathrm{p}=0.05)$ & 2.95 & 1.76 & 2.70 & 0.91 & 1.22 & 0.89 \\
\hline
\end{tabular}

*data transformed using square root transformation; figures in parentheses denote original values

A1 - Dipping with hole; A2 - Dipping without hole; A3 - Spraying; B1 - Fipronil 3.5\% (Thripguard 35 SC ${ }^{\mathrm{TM}}$ );

B2 - Imidacloprid 35\% (Confidor 350 SC ${ }^{\mathrm{TM}}$ ); B3 - The control (water) 
Also, with regard to the dipping method interaction, the effects reveal that Fipronil recorded the best larval mortality (100\% for small and medium larvae), while larval mortality with Imidacloprid ranged from $40-80 \%$. All larvae in the control (water) treatment were alive.

Results pertaining to the reduction of the dipping time as well as the lethal concentration showed that (Table 2; Fig. 1) Fipronil $0.004 \%$ gave $100 \%$ mortality at both the 60 and 30 min dippings of infested offshoots Furthermore,
$100 \%$ mortality of RPW larvae was also obtained when offshoots were dipped in Fipronil 0.002\% for $60 \mathrm{~min}$.

With regard to residual toxicity studies, $100 \%$ mortality of all the stages tested was obtained with $0.008 \%$ Fipronil using the dipping method (Table 3). The residual toxicity studies further supported the above results. The indication was, that the dipping of date palm offshoots for six hours in $0.008 \%$ Fipronil would give the necessary protection against both the larvae and adults of red palm

Table 2. Mortality of R. ferrugineus larvae in date palm offshoots dipped in varying concentrations of Fipronil 3.5\% (Thripguard 35 SC ${ }^{\mathrm{TM}}$ ) for different time intervals

\begin{tabular}{|c|c|c|c|}
\hline \multirow{2}{*}{ Treatment } & \multicolumn{3}{|c|}{ Mean larval mortality [\%] ${ }^{*}$} \\
\hline & trial-i & trial-ii & cumulative analysis \\
\hline \multicolumn{4}{|c|}{ Factor A: Concentration of Fipronil } \\
\hline A1 & $\begin{array}{c}9.81 \\
(96.00)\end{array}$ & $\begin{array}{c}9.66 \\
(93.33)\end{array}$ & $\begin{array}{c}9.74 \\
(94.67)\end{array}$ \\
\hline A2 & $\begin{array}{c}8.00 \\
(68.00)\end{array}$ & $\begin{array}{c}7.86 \\
(66.67)\end{array}$ & $\begin{array}{c}7.96 \\
(67.33)\end{array}$ \\
\hline A3 & $\begin{array}{c}6.30 \\
(41.00)\end{array}$ & $\begin{array}{c}5.25 \\
(29.33)\end{array}$ & $\begin{array}{c}5.88 \\
(35.33)\end{array}$ \\
\hline $\mathrm{A} 4$ & $\begin{array}{c}2.74 \\
(12.00)\end{array}$ & $\begin{array}{c}1.98 \\
(6.67)\end{array}$ & $\begin{array}{c}2.47 \\
(8.67)\end{array}$ \\
\hline $\mathrm{CD}(\mathrm{p}=0.05)$ & 1.04 & 0.98 & 0.81 \\
\hline \multicolumn{4}{|c|}{ Factor B: Dipping time } \\
\hline B1 & $\begin{array}{c}7.47 \\
(65.00)\end{array}$ & $\begin{array}{c}6.98 \\
(60.00)\end{array}$ & $\begin{array}{c}7.27 \\
(62.50)\end{array}$ \\
\hline B2 & $\begin{array}{c}7.03 \\
(59.00)\end{array}$ & $\begin{array}{c}6.55 \\
(54.00)\end{array}$ & $\begin{array}{c}6.92 \\
(56.50)\end{array}$ \\
\hline B3 & $\begin{array}{c}5.63 \\
(39.00)\end{array}$ & $\begin{array}{c}5.04 \\
(33.00)\end{array}$ & $\begin{array}{c}5.35 \\
(35.50)\end{array}$ \\
\hline $\mathrm{CD}(\mathrm{p}=0.05)$ & 0.90 & 0.85 & 0.81 \\
\hline \multicolumn{4}{|c|}{ Interaction $(\mathrm{A} \times \mathrm{B})$} \\
\hline A1B1 & $\begin{array}{c}10.03 \\
(100.00)\end{array}$ & $\begin{array}{c}10.03 \\
(100.00)\end{array}$ & $\begin{array}{c}10.03 \\
(100.00)\end{array}$ \\
\hline A1B2 & $\begin{array}{c}10.03 \\
(100.00)\end{array}$ & $\begin{array}{c}10.03 \\
(100.00)\end{array}$ & $\begin{array}{c}10.03 \\
(100.00)\end{array}$ \\
\hline A1B3 & $\begin{array}{c}9.39 \\
(88.00)\end{array}$ & $\begin{array}{c}8.92 \\
(80.00)\end{array}$ & $\begin{array}{c}9.18 \\
(84.00)\end{array}$ \\
\hline A2B1 & $\begin{array}{c}10.03 \\
(100.00)\end{array}$ & $\begin{array}{c}10.03 \\
(100.00)\end{array}$ & $\begin{array}{c}10.03 \\
(100.00)\end{array}$ \\
\hline $\mathrm{A} 2 \mathrm{~B} 2$ & $\begin{array}{c}8.71 \\
(76.00)\end{array}$ & $\begin{array}{c}8.71 \\
(76.00)\end{array}$ & $\begin{array}{c}18.72 \\
(76.00)\end{array}$ \\
\hline A2B3 & $\begin{array}{c}5.26 \\
(28.00)\end{array}$ & $\begin{array}{c}4.90 \\
(24.00)\end{array}$ & $\begin{array}{c}5.13 \\
(26.00)\end{array}$ \\
\hline A3B1 & $\begin{array}{c}6.85 \\
(48.00)\end{array}$ & $\begin{array}{c}5.63 \\
(32.00)\end{array}$ & $\begin{array}{c}6.27 \\
(40.00)\end{array}$ \\
\hline A3B2 & $\begin{array}{c}6.80 \\
(48.00)\end{array}$ & $\begin{array}{c}6.00 \\
(36.00)\end{array}$ & $\begin{array}{c}6.46 \\
(42.00)\end{array}$ \\
\hline A3B3 & $\begin{array}{c}5.26 \\
(28.00)\end{array}$ & $\begin{array}{c}4.13 \\
(20.00)\end{array}$ & $\begin{array}{c}4.90 \\
(24.00)\end{array}$ \\
\hline A4B1 & $\begin{array}{c}3.00 \\
(12.00)\end{array}$ & $\begin{array}{c}2.24 \\
(8.00)\end{array}$ & $\begin{array}{c}2.74 \\
(10.00)\end{array}$ \\
\hline A4B2 & $\begin{array}{c}2.60 \\
(12.00)\end{array}$ & $\begin{array}{c}1.47 \\
(4.00)\end{array}$ & $\begin{array}{c}2.49 \\
(8.00)\end{array}$ \\
\hline A4B3 & $\begin{array}{c}2.60 \\
(12.00)\end{array}$ & $\begin{array}{c}2.24 \\
(8.00)\end{array}$ & $\begin{array}{c}8.00 \\
(2.18)\end{array}$ \\
\hline $\mathrm{CD}(\mathrm{p}=0.05)$ & 1.79 & 1.70 & 1.88 \\
\hline
\end{tabular}

*data transformed using square root transformation; figures in parentheses denote original values

A1 - Dipping offshoot in 0.004\% Fipronil 3.5\% (Thripguard 35 SC ${ }^{\mathrm{TM}}$ ); A2 - Dipping offshoot in 0.002\% Fipronil; A3 - Dipping offshoot in 0.001\% Fipronil; A4 - Dipping offshoot in water (the control); B1 - Dipping offshoot in Fipronil for 60 min, B2 - Dipping offshoot in Fipronil for 30 min, B3 - Dipping offshoot in Fipronil for 15 min 


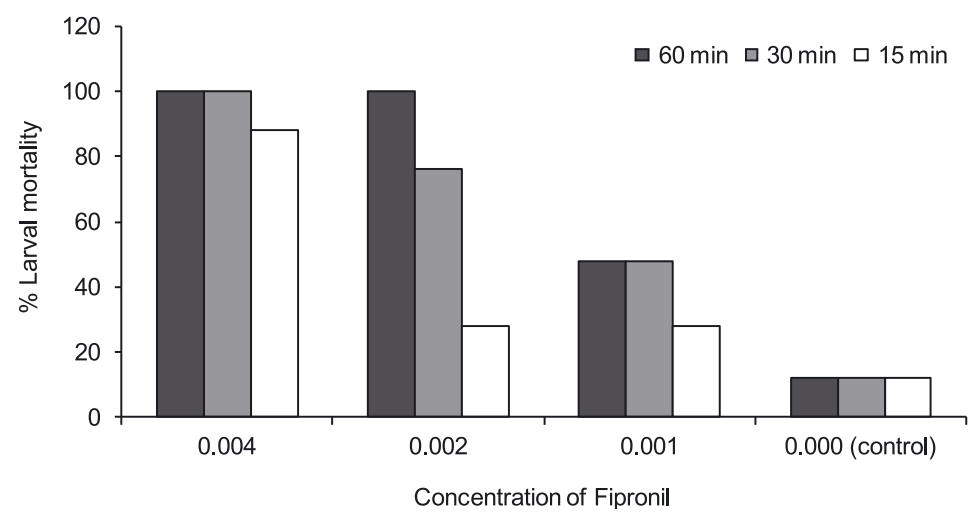

Fig. 1. Larval mortality of RWP infested date palm offshoots dipped in different concentrations of Fipronil for different durations

Table 3. Residual toxicity to R. ferrugineus, $72 \mathrm{~h}$ after dipping/24 h after spraying date palm offshoots in Fipronil $3.5 \%$ (Thripguard $35 \mathrm{SC}^{\mathrm{TM}}$ ) and Imidacloprid $35 \%$ (Confidor $350 \mathrm{SC}^{\mathrm{TM}}$ )

\begin{tabular}{|c|c|c|c|c|c|}
\hline \multirow{2}{*}{ Treatments } & \multicolumn{5}{|c|}{ Mean percent mortality* } \\
\hline & small larvae & medium larvae & large larvae & adults (male) & adults (female) \\
\hline \multicolumn{6}{|c|}{ Factor A: Treatment method (Dipping/Spraying) } \\
\hline A1 & $\begin{array}{c}6.92 \\
(66.67)\end{array}$ & $\begin{array}{c}6.92 \\
(66.67) \\
\end{array}$ & $\begin{array}{c}4.43 \\
(40.00)\end{array}$ & $\begin{array}{c}6.30 \\
(60.00)\end{array}$ & $\begin{array}{c}4.43 \\
(40.00)\end{array}$ \\
\hline $\mathrm{A} 2$ & $\begin{array}{c}6.92 \\
(66.67)\end{array}$ & $\begin{array}{c}6.92 \\
(66.67)\end{array}$ & $\begin{array}{c}5.06 \\
(46.67) \\
\end{array}$ & $\begin{array}{c}4.43 \\
(40.00)\end{array}$ & $\begin{array}{c}5.06 \\
(46.67) \\
\end{array}$ \\
\hline A3 & $\begin{array}{c}0.71 \\
(0.00) \\
\end{array}$ & $\begin{array}{c}2.57 \\
(20.00) \\
\end{array}$ & $\begin{array}{c}1.33 \\
(6.67) \\
\end{array}$ & $\begin{array}{c}1.95 \\
(13.33) \\
\end{array}$ & $\begin{array}{c}1.33 \\
(6.67) \\
\end{array}$ \\
\hline $\mathrm{CD}(\mathrm{p}=0.05)$ & 1.59 & 1.27 & 1.93 & 2.15 & 1.93 \\
\hline \multicolumn{6}{|c|}{ Factor B: Insecticide (Fipronil/Imidacloprid/The control) } \\
\hline B1 & $\begin{array}{c}9.40 \\
(93.33)\end{array}$ & $\begin{array}{c}8.78 \\
(86.67)\end{array}$ & $\begin{array}{c}7.54 \\
(73.33) \\
\end{array}$ & $\begin{array}{c}7.54 \\
(73.33)\end{array}$ & $\begin{array}{c}6.92 \\
(66.67)\end{array}$ \\
\hline B2 & $\begin{array}{c}8.16 \\
(8.00) \\
\end{array}$ & $\begin{array}{c}6.92 \\
(66.67)\end{array}$ & $\begin{array}{c}2.57 \\
(20.00)\end{array}$ & $\begin{array}{c}3.81 \\
(33.33)\end{array}$ & $\begin{array}{c}3.19 \\
(26.67) \\
\end{array}$ \\
\hline B3 & $\begin{array}{c}0.71 \\
(0.00) \\
\end{array}$ & $\begin{array}{c}0.71 \\
(0.00) \\
\end{array}$ & $\begin{array}{c}0.71 \\
(0.00) \\
\end{array}$ & $\begin{array}{c}1.33 \\
(6.67) \\
\end{array}$ & $\begin{array}{c}0.71 \\
(0.00) \\
\end{array}$ \\
\hline $\mathrm{CD}(\mathrm{p}=0.05)$ & 1.59 & 1.27 & 1.93 & 2.15 & 1.93 \\
\hline \multicolumn{6}{|c|}{ Interaction } \\
\hline A1B1 & $\begin{array}{c}10.03 \\
(100.00)\end{array}$ & $\begin{array}{c}10.03 \\
(100.00)\end{array}$ & $\begin{array}{c}10.03 \\
(100.00)\end{array}$ & $\begin{array}{c}10.03 \\
(100.00)\end{array}$ & $\begin{array}{c}10.03 \\
(100.00)\end{array}$ \\
\hline A1B2 & $\begin{array}{c}10.03 \\
(100.00)\end{array}$ & $\begin{array}{c}10.03 \\
(100.00)\end{array}$ & $\begin{array}{c}2.57 \\
(20.00)\end{array}$ & $\begin{array}{c}8.16 \\
(80.00)\end{array}$ & $\begin{array}{c}2.57 \\
(20.00)\end{array}$ \\
\hline A1B3 & $\begin{array}{c}0.00 \\
(0.71) \\
\end{array}$ & $\begin{array}{c}0.00 \\
(0.71) \\
\end{array}$ & $\begin{array}{c}0.71 \\
(0.00) \\
\end{array}$ & $\begin{array}{c}0.71 \\
(0.00) \\
\end{array}$ & $\begin{array}{c}0.71 \\
(0.00) \\
\end{array}$ \\
\hline A2B1 & $\begin{array}{c}10.03 \\
(100.00) \\
\end{array}$ & $\begin{array}{c}10.03 \\
(100.00) \\
\end{array}$ & $\begin{array}{c}10.03 \\
(100.00)\end{array}$ & $\begin{array}{c}10.23 \\
(100.00) \\
\end{array}$ & $\begin{array}{c}10.03 \\
(100.00)\end{array}$ \\
\hline A2B2 & $\begin{array}{c}10.03 \\
(100.00)\end{array}$ & $\begin{array}{c}10.03 \\
(100.00)\end{array}$ & $\begin{array}{c}4.43 \\
(40.00)\end{array}$ & $\begin{array}{c}2.57 \\
(20.00)\end{array}$ & $\begin{array}{c}4.43 \\
(40.00)\end{array}$ \\
\hline A2B3 & $\begin{array}{c}0.00 \\
(0.71) \\
\end{array}$ & $\begin{array}{c}0.00 \\
(0.71) \\
\end{array}$ & $\begin{array}{c}0.71 \\
(0.00) \\
\end{array}$ & $\begin{array}{c}0.71 \\
(0.00) \\
\end{array}$ & $\begin{array}{c}0.71 \\
(0.00) \\
\end{array}$ \\
\hline A3B1 & $\begin{array}{c}8.16 \\
(80.00) \\
\end{array}$ & $\begin{array}{c}8.16 \\
(80.00) \\
\end{array}$ & $\begin{array}{c}2.57 \\
(20.00) \\
\end{array}$ & $\begin{array}{c}2.57 \\
(20.00) \\
\end{array}$ & $\begin{array}{c}0.71 \\
(0.00)\end{array}$ \\
\hline A3B2 & $\begin{array}{c}4.43 \\
(40.00)\end{array}$ & $\begin{array}{c}0.00 \\
(0.71)\end{array}$ & $\begin{array}{c}0.71 \\
(0.00)\end{array}$ & $\begin{array}{c}0.71 \\
(0.00)\end{array}$ & $\begin{array}{c}2.57 \\
(20.00)\end{array}$ \\
\hline A3B3 & $\begin{array}{c}0.00 \\
(0.71)\end{array}$ & $\begin{array}{c}0.00 \\
(0.71)\end{array}$ & $\begin{array}{c}0.71 \\
(0.00) \\
\end{array}$ & $\begin{array}{c}2.57 \\
(20.00)\end{array}$ & $\begin{array}{c}0.00 \\
(0.00)\end{array}$ \\
\hline $\mathrm{CD}(\mathrm{p}=0.05)$ & 2.76 & 1.79 & 3.35 & 3.72 & 3.48 \\
\hline
\end{tabular}

*data transformed using square root transformation; figures in parentheses denote original values A1 - Dipping with hole; A2 - Dipping without hole; A3 - Spraying; B1 - Fipronil 3.5\% (Thripguard 35 SC ${ }^{\mathrm{TM}}$ ); B2 - Imidacloprid 35\% (Confidor $350 \mathrm{SC}^{\mathrm{TM}}$ ); B3 - The control (water) 
weevil. Drilling of holes into offshoots before dipping in insecticide did not significantly enhance larval and adult mortality and is therefore, not required to be incorporated into the quarantine protocol. It is pertinent to mention, that similar residual toxicity tests with date palm offshoots dipped in Fipronil $0.004 \%$ for 30 min, resulted in $100 \%$ mortality of newly emerged, small, medium, and large larvae, besides resulting in complete mortality of adult male and female weevils. There have been no phytotoxic symptoms recorded in any of the ten offshoots treated with the above protocol (Fipronil $0.004 \%$ for $30 \mathrm{~min}$ ) for one year. All treated offshoots with the recommended protocol established well in the field.

\section{DISCUSSION}

In the trial pertaining to the method of treatment (dipping/spraying of offshoots) and choice of insecticide to kill hidden larvae of RPW in date palm offshoots, dipping was found to be better than spraying. Fipronil recorded a $100 \%$ mortality for both small and medium sized RPW larvae as compared to Imidacloprid where larval mortality ranged from $40-80 \%$ in the dipping method. Previously, Fipronil was reported recording the least $\mathrm{LC}_{50}$ values against both adults and larvae of RPW among eight tested insecticides, indicating the high level of toxicity against this pest (Al-Shawaf et al. 2010). Barranco et al. 1998, through insecticide laboratory assays, found that more than $0.1 \mathrm{ppm}$ of Fipronil incorporated in a semi artificial diet resulted in 100\% mortality of young larvae. Complete mortality of 30 day old RPW larvae with Fipronil at more than 0.2 ppm was reported by Abdulsalam et al. 2001, thus making it a choice candidate for insecticide based quarantine protocols against this pest. Semi-field trials with Imidacloprid applied by soil injection in preventive and curative treatments to $P$. canariensis in Spain showed $100 \%$ and $94 \%$ efficacies, respectively (Llácer et al. 2012). Further, in a field assay against RPW, two applications of Imidacloprid per year successfully reduced mortality of $P$. canariensis palms to less than $27 \%$ compared to more than $84 \%$ for untreated control palms (Dembilio et al. 2010).

Previous reports from Saudi Arabia and Israel recommended that the base of date palm offshoots be dipped in insecticide solution/mud-insecticide mixtures before they are transported elsewhere for planting as a quarantine measure (Anonymous 1998; Anonymous 2004). The preferred insecticide, its strength, and duration of treatment, however, were not known. Our study indicated that, Fipronil $0.004 \%$ gave $100 \%$ mortality at both 60 and 30 min dipping of infested offshoots. Furthermore, 100\% mortality of RPW larvae was also obtained when offshoots were dipped in Fipronil $0.002 \%$ for $60 \mathrm{~min}$.

Abdulsalam et al. 2001 reported that Fipronil was effective in suppressing adult emergence from pupae. Fipronil 50 Sc and 200 Sc achieved 100\% mortality of larvae and adults at $25 \mathrm{ppm}$ after one week. In our study, results pertaining to residual toxicity showed $100 \%$ mortality of all the stages tested including newly emerged larvae, obtained by the dipping method $72 \mathrm{~h}$ after treatment (0.004\% Fipronil for $30 \mathrm{~min}$ ).
Studies carried out in Spain in P. canariensis suggest that a dose of $1.14 \mathrm{~g}$ aluminium phosphide $/ \mathrm{m}^{3}$ for 3 days is enough to kill all the stages of RPW in an infested palm tree, and is recommended as a quarantine protocol provided the dose is not phytotoxic to the treated palms (Llácer and Jacas 2010). No phytotoxic symptoms have been recorded in any of the ten offshoots treated with the above protocol (Fipronil $0.004 \%$ for $30 \mathrm{~min}$ ) and planted in the field for one year after planting.

Sounding devices have been used to detect RPW larvae feeding inside the palm (Soroker et al. 2004). These devices, though, need further refinement to attain better detection accuracy and eliminate the possibility of newly emerged RPW larvae going undetected. The insecticide based quarantine protocol of dipping date palm offshoots in $0.004 \%$ Fipronil for $30 \mathrm{~min}$ developed in the study, will help ensure movement of date palm offshoots free of RPW larvae besides protecting the treated offshoots from invading adults. This protocol can be implemented by the concerned authorities in date palm growing countries of the Middle East and elsewhere. The risk of concealed RPW larvae moving through date palm offshoots transported for planting will then be minimized. In addition to the quarantine protocol developed in this study for date palm offshoots, there is a need to develop a quarantine protocol against RPW for bigger date palms that are shipped/transported for ornamental landscape gardening and also to implement a strict regulatory mechanism of treatment, certification and monitoring of palms for planting (either for farming or ornamental gardening) based on the model of the European Union (EU 2007) where the palm trade is strictly regulated due to the threat of RPW.

\section{ACKNOWLEDGEMENT}

The authors thank the Ministry of Agriculture, Kingdom of Saudi Arabia for providing the necessary facilities to conduct this study at the Date Palm Research Centre (DPRC), Al-Hassa, through the FAO funded project UTFN/SAU/043/SAU. We also wish to thank the Director, DPRC for the support extended in carrying out the study.

\section{REFERENCES}

Abraham V.A., Al Shuaibi M., Faleiro J.R., Reda A. Abozuhairah, Vidyasagar P.S.P.V. 1998. An integrated approach for the management of red palm weevil Rhynchophorus ferrugineus Oliv. - a key pest of date palm in the Middle-East. Sultan Qaboos Univ. J. Sci. Res. 3: 77-83.

Abdulsalam K.S., Shawir M.S., Abo-El- Saad M.M., Rezk M.A., Ajlan A.M 2001. Regent (fipronil) as a candidate insecticide to control Red Palm Weevil (Rhynchophorus ferrugineus Oliv.). Ann. Agric. 46 (2): 841-849.

Al-Shawaf Abdul Moneim A., Al-Shagagh Abdallah A., Al-Bakshi M.M., Al-Saroj S.A., Al-Badr S.M., Al-Dandan A.M., Ben Abdallah A. 2010. Toxicity of some insecticides against Red Palm Weevil Rhynchophorus ferrugineus (Coleoptera: Curculionidae). Indian J. Plant Prot. 38 (1): 13-16. 
Anonymous 1998. Final report of the Indian technical team. Part A - Red Palm Weevil Control Project. Ministry of Agriculture and Water, Kingdom of Saudi Arabia, 65 pp.

Anonymous 2004. The Middle East Red Palm Weevil Programme. July 1998 to June 2004. Final report. The Peres Center for Peace, Jaffa, Israel, 62 pp.

Anonymous 2013. Save algarve palms. http://www.savealgarvepalms.com/en/weevil-facts/host-palm-trees [Accessed: March 24, 2013].

Barranco P., Pena J.A. De la, Martin M.M., Cabell T. 1998. Efficacy of chemical control of the new palm pest Rhynchophorus ferrugineus (Olivier 1790) (Curculionidae: Coleoptera). Bol. de Sanidad Veg. Plagas 24 (2): 301-306.

Dembilio O., Jacas J.A., Llácer E. 2009. Are the palms Washingtonia filifera and Chamaerops humilis suitable hosts for the red palm weevil, Rhynchophorus ferrugineus (Coleoptera: Curculionidae)? J. Appl. Entomol. 133 (1): 565-567.

Dembilio O., Llacer E., Martinez de Altube M.M., Jacas J.A. 2010. Field efficacy of imidacloprid and Steinernema carpocapsae in a chitosan formulation against the red palm weevil Rhynchophorus ferrugineus (Coleoptera: Curculionidae) in Phoenix canariensis. Pest Manage. Sci. 66 (4): 365-370.

EU (European Union) 2007. Commission decision of 25 May 2007 on emergency measures to prevent the introduction into and the spread within the Community of Rhynchophorus ferrugineus (Olivier). 2007/365/EC. Official J. European Union 139: 24-27.

EPPO Reporting Service 2009. Rhynchophorus ferrugineus found on Howea forsteriana in Sicilia.No. 12, 2009/051, Paris, 39 pp.

El-Sabea A.M.R., Faleiro J.R., Abo El-Saad M.M. 2009. The threat of red palm weevil Rhynchophorus ferrugineus to date plantations of the Gulf region of the Middle-East: an economic perspective. Outlook on Pest Manage 20 (3): 131-134.

Fiaboe K.K.M., Peterson A.T., Kairo M.T.K., Roda A.L. 2012. Predicting the potential worldwide distribution of the red palm weevil Rhynchophorus ferrugineus (Olivier) (Coleoptera: Curculionidae) using ecological niche modeling. Fl. Entomol. 95 (3): 559-673.

FAOstat 2012. http://faostat.fao.org/ [Accessed: March 11, 2013].

Faleiro J.R. 2006. A review of the issues and management of red palm weevil Rhyncophorus ferrugineus (Coleoptera: Rhynchophoridae) in coconut and date palm during the last one hundred years. Int. J. Tropical Ins. Sci. 26 (3): 135-154.
Llácer E., Jacas J.A. 2010. Efficacy of phosphine as a fumigant against Rhynchophorus ferrugineus (Coleoptera: Curculionidae) in palms. Spanish J. Agric. Res. 8 (3): 775-779.

Llácer E., Negre M., Jacas J.A. 2012. Evaluation of an oil dispersion formulation of imidacloprid against Rhynchophorus ferrugineus (Coleoptera, Curculionidae) in young palm trees. Pest Manage. Sci. 68 (6): 878-882.

Malumphy C., Moran H. 2009. Red palm weevil, Rhynchophorus ferrugineus. Plant Pest Fact Sheet. http://www.fera.defra.gov.uk/plants/publications/factsheets/redpalmweevil [Accessed: February 19, 2012].

Mousavi M., Mousavi A., Habashi A.A. Arzani K. 2009. Optimization of physical and biological parameters for transient expression of uidA gene in embryogenic callus of date palm (Phoenix dactylifera L.) via particle bombardment. African J. Biotechnol. 8 (6): 3721-3730.

OJEU 2008. Commission decision of 6 October 2008 amending decision of 2007/365/EC on emergency measures to prevent the introduction into and the spread within the community of Rhynchophorus ferrugineus (Olivier) 2008/5550/C. Official J. European Union L 266: 14.

Oehlschlager A.C. 2005. Current status of trapping palm weevils and beetles. The Planter 81 (947): 123-143.

Salama H.S., Abd-Elgawad M.M. 2003. Quarantine problems: an analytical approach with special reference to palm weevils and phytonematodes. Archiv. Phytopathol. Plant Prot. 36 (1): 41-46.

Sallam A.A., El-Shafie H.A.F., Al-Abdan S. 2012. Influence of farming practices on infestation by red palm weevil Rhynchophorus ferrugineus (Olivier) in date palm: a case study. Int. Res. J. Agric. Sci. Soil Sci. 2 (8): 370-376.

Soroker V., Nakache Y., Landau U., Mizrach A., Hetzroni A., Gerling D. 2004. Utilization of sounding methodology to detect infestation by Rhynchophorus ferrugineus on palm offshoots. Phytoparasitica 33 (1): 6-8.

Tomlin C.D.S. 2011. The Pesticide Manual: A World Compendium. 15th ed. CAB International, Wallingford, Oxon, UK, 1457 pp.

Zaid A., De Wet P.F., Djerbi M., Oihabi A. 2002. Diseases and pests of date palm (Chapter XII). p. 227-281. In: “Date Palm Cultivation" (A. Zaid, ed.). FAO Plant Prod. Prot. Paper 156, Rome, Italy. 